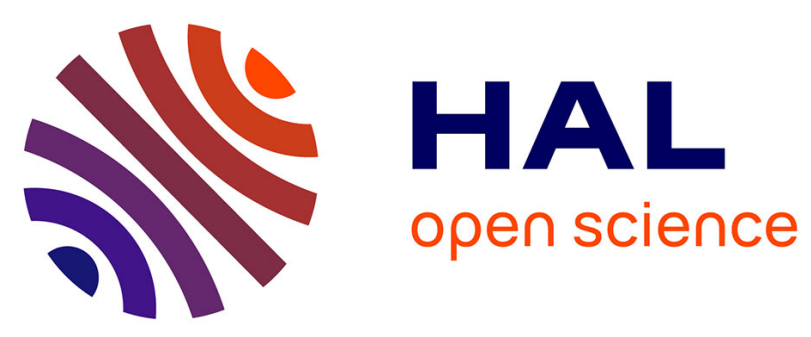

\title{
Modélisation des avalanches
}

Gérard Brugnot

\section{To cite this version:}

Gérard Brugnot. Modélisation des avalanches. Revue forestière française, 1982, 34 (5), pp.131-142. 10.4267/2042/21592 . hal-03423436

\section{HAL Id: hal-03423436 https://hal.science/hal-03423436}

Submitted on 10 Nov 2021

HAL is a multi-disciplinary open access archive for the deposit and dissemination of scientific research documents, whether they are published or not. The documents may come from teaching and research institutions in France or abroad, or from public or private research centers.
L'archive ouverte pluridisciplinaire HAL, est destinée au dépôt et à la diffusion de documents scientifiques de niveau recherche, publiés ou non, émanant des établissements d'enseignement et de recherche français ou étrangers, des laboratoires publics ou privés. 


\section{MODÉLISATION DES}

\section{AVALANCHES}

\section{G. BRUGNOT}

On rencontre dans l'étude des avalanches, la même dichotomie que dans l'étude d'autres phènomènes dynamiques naturels.

D'une part. se pose la question des conditions d'apparition du phénomène qui introduit toutes les recherches relatives à la stabilité d'un milieu. Dans le cas de la neige. il s'agit d'un matériau très complexe qui ne peut s'analyser de façon purement mécanique. En effet. la neige est l'objet de transformations de type thermodynamique qui affectent beaucoup sa stabilité, que celle-ci soit considérée en un point ou à l'échelle d'un versant. L'étude de la stabilité du manteau neigeux repose donc sur une étude détaillée de la physique du cristal de glace. puis de la neige constituée de ce cristal situe dans un environnement bien particulier. De telles considérations ont attiré de nombreux chercheurs. ce qui s'explique par une double particularité : il s'agit d'un domaine complique sur le plan théorique. mais certaines vérifications expérimentales sont relativement faciles à opèrer. Enfin. il n'est pas contestable qu'un grand interêt pratique justitie ces recherches. puisque les divers usagers de la montagne sont tous intéressés par une prévision fiable du risque d'avalanche.

D'autre part, on est confronté au sujet, non moins important pratiquement, de la dynamique du phénomène et là. il faut bien reconnaître que la "recherche est beaucoup moins active.

D'où résulte ce manque d'intérêt? Je pense que nous sommes en présence d'un domaine qui est moins propice à des considerations théoriques fondamentales. tandis que les mesures sont très compliquées: on ne peut donc que difficilement vérifier des hypothèses qui elles-mêmes sont assez hasardeuses. Ce qui est vrai pour la neige l'est aussi pour d'autres phénomènes dynamiques naturels comme celui des glissements de terrain.

Cependant. l'enjeu pratique est considérable. D'une part, il s'agit de dessiner avec la plus grande précision possible le contour des zones exposées à risque. D'autre part. il faut être en mesure de calculer des ouvrages de protection qui vont être soumis aux eftets dynamiques exercès par l'avalanche. 


\section{G. BRUGNOT}

Tout ceci a incité le C.T.G.R.E.F., devenu rẻcemment le C.E.M.A.G.R.E.F., à entreprendre diverses études de dynamiques des avalanches pour le compte notamment des services de Restauration des Terrains en Montagne qui sont fréquemment sollicités par les collectivités locales dans le domaine de la protection contre les avalanches.

Je décrirai dans ce qui suit les actions de recherche poursuivies dans le domaine de la modèlisation des avalanches, en insistant sur celles qui sont menées en France. Depuis le début, la division "Nivologie" du C.T.G.R.E.F. a été au centre de ces recherches qui, après certaines tentatives entreprises à la tin des années 60 , ont reçu une impulsion incontestable de l'accident de février 1970 survenu à Val-d'Isère en Savoie; cet accident a, on le sait, conduit à la création de la division "Nivologie" au C.T.G.R.E.F.

\section{LES DEUX GRANDS TYPES D'AVALANCHES}

Du point de vue de la dynamique du phénomène, on peut classer les avalanches en 2 types, que nous déciderons d'appeler "avalanche de neige dense " et " avalanche de neige poudreuse ".

\section{L'avalanche de neige dense}

Elle suit à peu près les mêmes lois qu'un écoulement hydraulique torrentiel. C'est un écoulement non permanent, que l'on peut rapprocher de celui qui fait suite à la rupture d'un barrage hydraulique. Le fait important est que la densitè de la neige dans l'écoulement reste du même ordre de grandeur que la densité de la neige en place, à partir de laquelle s'est formée l'avalanche. On verra que lorsqu'il atteint une certaine vitesse, l'écoulement de neige dense est fortement turbulent, mais avant ce stade (zone de départ) et après (zone d'arrêt), il traverse des stades de frottement laminaire et de frottement solide. Dans le cadre de cet exposé, on peut classer tous ces phénomènes dans la même rubrique puisque, on le verra, ils peuvent être représentés par le même modèle.

\section{L'avalanche de neige poudreuse}

Elle se développe à partir d'une avalanche de neige dense. Quand cette avalanche atteint un régime turbulent assez rapide (vers $20 \mathrm{~m} / \mathrm{s}$ ), le trottement ècoulement-air commence à exercer un rôle sur la dynamique de l'écoulement, l'avalanche a tendance à "gonfler " par incorporation d'air ambiant et si certaines conditions sont réunies (pente longue et raide, neige sans cohésion), on assiste à l'apparition de neige poudreuse, sorte de nuage s'alimentant à l'avant par prélévement sur le manteau neigeux en place, tandis qu'à l'arrière il y a également alimentation par un écoulement stationnaire qui suit le nuage. Une avalanche poudreuse peut atteindre une vitesse de l'ordre de $100 \mathrm{~m} / \mathrm{s}$, mais alors sa densitè est plus proche de l'air ambiant que de celle de la neige à partir de laquelle elle s'est formée.

\section{Conditions de formation}

Il est ditficile de donner des critères précis déterminant l'apparition d'avalanche d'un type ou d'un autre. Ce qui est à peu près certain, c'est que l'avalanche de neige poudreuse ne peut se former qu'à partir de neige récente, à la fois très froide et de faible densité (les deux sont corrèlès). En outre, on peut supposer que certaines conditions d'échelle doivent être réunies: surface importante de la zone de départ, c'est-à-dire de la neige mise en mouvement, longue distance d'ècoulement avec, en outre, un tacteur favorable qui est celui des accidents de terrain, tels barres rocheuses, virage brutal de la zone d'ècoulement, etc. 


\section{Les avalanches}

\section{MOdÉlisation des aVALANCHES DE NEIGE DENSE}

On a donc donné cette appellation commune à des écoulements divers, mais ces écoulements peuvent être calculès par le même modèle numérique, car les forces qui interviennent sont les mêmes :

$$
\begin{array}{ll}
\text { - pesanteur, } & - \text { frottement au sol, } \\
\text { - forces de pression, } & - \text { inertie. }
\end{array}
$$

En réalité, le terme de frottement peut intégrer des termes: turbulent, laminaire et solide, ce qui permet de simuler en fait trois types d'écoulement différents, qui correspondent à des phases successives du phénomène:

solide $\rightarrow$ laminaire $\rightarrow$ turbulent $\rightarrow$ laminaire $\rightarrow$ solide.

A partir de ces considérations fondamentales, on a construit plusieurs modèles, ceux-ci se classant en 2 grandes categories, les modèles simplifiés et les modèles numériques au sens propre du terme.

\section{Les madèles simplifiés. Le modèle de Voellmy}

Ces modèles ne représentent en fait que le mouvement du centre de gravité de l'avalanche. On prend donc en compte la pesanteur et l'inertie et, parmi les frottements, le frottement sec et/ou le frottement laminaire et/ou le frottement turbulent. En réalité, il semble que les premiers modèles connus ont été proposés par des chercheurs soviétiques, avant la Deuxième Guerre mondiale, mais ils ne contenaient pas de terme de frottement turbulent (Goff et Otten, 1939).

C'est pour cette raison que la science des avalanches a conservé plutôt le nom de Voellmy qui, dans un article paru en 1950, a proposé un modèle avec frottement turbulent. II a obtenu ainsi une formule tout à fait analogue à celle de Chezy en hydraulique. Par exemple, dans le cas d'une avalançhe canalisée:

$$
\begin{aligned}
& \text { Force active : pesanteur } \quad M g \sin \alpha\left(^{*}\right) \\
& \text { Force passive : inertie } \quad M \frac{d v}{d t} \\
& \text { frottement solide } \quad r M g \cos \alpha \\
& \text { frottement turbulent } \varrho \mathrm{BL} \frac{v^{2}}{\xi}
\end{aligned}
$$

La formule de Voellmy peut être naturellement critiquée du fait de sa simplification et de ses omissions, mais son succès est dû, à mon avis, à ce qu'elle représente le modèle le plus complexe qui puisse être résolu de façon analytique. Si on égale à 0 les forces décrites ci-dessus, on aboutit à une équation différentielle liant le carré de la vitesse à l'abscisse curviligne s. La solution de cette équation est du type:

$$
v^{2}=\left(v_{1}\right)^{2}\left[1-\operatorname{ex}\left(-\frac{2 g}{\xi} \frac{s}{h}\right)\right]\left(^{*}\right)
$$

\footnotetext{
( $\left.{ }^{*}\right)$ : angle de la pente (constant).

$M$ : masse de l'avalanche.

B: périmétre mouillè par l'avalanche.

$\checkmark$ : vitesse de l'avalanche (centre de gravité).

$\mathrm{g}$ : accèlération de la pesanteur.

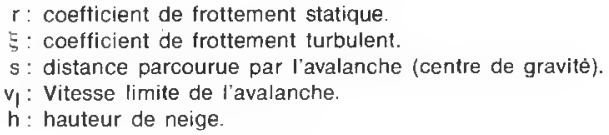

$r$ : coefticient de frottement statique.

5 : coefficient de frottement turbulent.

$\mathrm{s}$ : distance parcourue par l'avalanche (centre de gravitè).

$v_{1}$ : Vitesse limite de l'avalanche.

$h$ : hauteur de neige 


\section{G. BRUGNOT}

Le modèle de Voellmy conduit donc au résultat suivant: sur une pente constante. on atteint une vitesse limite $V_{1}$ telle que:

$$
\left(v_{1}\right)^{2}=\xi h(\sin \alpha-r \cos \alpha)
$$

Cette vitesse limite est atteinte rapidement, car dans l'exponentielle écrite ci-dessus le coefficient $\mathrm{g} / 5$ est de l'ordre de $10^{-2}$. Comme $h$ est de l'ordre du mètre. on est environ au deux tiers de la vitesse limite dès que le centre de gravité de l'avalanche a parcouru environ $100 \mathrm{~m}$. II faut remarquer que $h$ est la hauteur de l'écoulement, qui est inconnue, tout en étant de l'ordre de grandeur de la hauteur de neige partie. tant qu'il n'y a pas une trop grande concentration par le relief. D'autres auteurs ont essayé de lier leur nom à celui d'un modèle, mais ils se sont contentés de changer des coefficients au modèle de Voellmy, en compliquant la résolution de l'équation.

\section{Les modèles numériques proprement dits. Le modèle C.E.M.A.G.R.E.F.}

Les imperfections du modèle Voellmy sont évidentes, mais si l'on veut se rapprocher de la réalité. par exemple prendre en considération le mouvement de l'avalanche et non de son seul centre de gravité pour avoir ce qui nous intéresse le plus. c'est-à-dire la vitesse du front avant; si l'on veut tenir compte de la force d'inertie liée à la reprise de neige en place. mécanisme fondamental pour expliquer la croissance de l'avalanche; si l'on veut intégrer la complexité du relief etc. on est rapidement affronté à des équations difficiles à manipuler et en tout cas insolubles analytiquement.

Dans ce domaine, ce sont également les Soviétiques qui sont allés le plus loin mais, quoique très intéressants, les résultats auxquels ils sont arrivés étaient purement qualitatifs (Koulikovsky et Eglite. 1973).

Ce n'est qu'avec l'apparition de moyens de calcul de plus en plus puissants que le calcul numérique a pu affronter la résolution des équations fondamentales de l'hydraulique dans le cas de conditions aux limites plus ou moins particulières. Si l'on définit en effet un écoulement par les forces en jeu et les conditions aux limites. on s'aperçoit que l'avalanche dense peut être assimilée à l'écoulement hydraulique résultant de la rupture d'un barrage. Le rapprochement est assez rigoureux à deux restrictions près. D'une part, l'avalanche est un écoulement compressible, quoique dans le cas de l'avalanche dense, sa densite reste voisine de celle de la neige en place; et on ne connaît pas de loi reliant la densite aux autres grandeurs caractéristiques de l'écoulement. D'autre part, il est difficile d'éliminer une certaine incertitude au sujet de la reprise de matériaux par l'écoulement, mais ceci est aussi vrai pour le modèle hydraulique.

C'est en France, grâce à une collaboration très étroite entre la division "Nivologie " et la division "Hydrologie-Hydraulique fluviale" que le premier modèle de calcul numérique d'avalanche dense a été mis au point (Carry et Pochat, 1978). Parallèlement une modélisation du même genre a été réalisée aux États-Unis, par I'Université du Montana, pour le compte du Forest Service (Lang, Dawson et Martinelli, 1979).

Le principe et l'intérêt d'un modèle numérique est que la limite des possibilités de réalisation n'est plus la capacité de calcul, mais la mauvaise connaissance des mécanismes fondamentaux. Selon les cas, cette méconnaissance pourra être plus ou moins accentuée. Par exemple. on est certain de la formulation d'une loi de frottement. mais il reste à déterminer les coefficients de cette loi. A l'opposé, on peut tout ignorer d'un mécanisme sur lequel on ne peut que faire diverses hypothèses; c'est le cas de la reprise de la neige à l'avant de l'avalanche.

Il en résulte que l'utilisation de l'ordinateur ne permet pas de "calculer" complètement une avalanche. Il s'agit plutôt, en un premier temps, de disposer d'un outil de recherche et c'est 
en "calant" un modèle sur des opérations de terrain que l'on peut espérer progresser dans la connaissance du phénomène. L'aspect physique de cette recherche ne doit pas complètement disparaître au profit de la mécanique de calcul

Concrètement. que nous permet de prendre en compte le modèle numérique?

Dans quelle mesure nous permet-elle de mieux approcher la réalité ?

Pour répondre à cette question. on doit décrire les équations du modèle. Ce sont les équations de I'hydraulique caractérisant un écoulement avec ressaut mobile. Par exemple. en utilisant les notations de Brugnot et Pochat. 1981:

$$
\begin{aligned}
& \frac{\partial S}{\partial t}+\frac{\partial P}{\partial x}=0 \quad(1)\left(^{*}\right) \\
& \frac{\partial P}{\partial t}-2 \frac{P}{S} \frac{\partial S}{\partial t}+\left(\frac{[g h]}{n}-\frac{P^{2}}{S^{2}}\right) \frac{\partial S}{\partial x}= \\
& =g S \sin \psi+\frac{g h}{n}\left(\frac{\partial S}{\partial x}\right)_{h=c o n s t a n t e}-\left(f_{s} g S \cos \psi+f_{1} g \frac{P}{R^{2}}+f_{t} \frac{P^{2}}{S R}\right) \\
& W\left(S-S_{0}\right)=P \quad(3) \quad \text { (4) } \\
& P(w-v)=\frac{g}{n+1}\left(S h-S_{0} h_{0}\right)
\end{aligned}
$$

Ces équations correspondent successivement à la conservation de la matière dans l'avalanche (1). à la conservation de la quantité de mouvement dans l'avalanche (2), à la conservation de la matière au front (3) et à la conservation de la quantité du mouvement du front (4) : équation d'un ressaut mobile.

Par rapport au modèle simplifié de type Voellmy. quel progrès représente donc ce modèle numérique? D'abord. on represente l'avalanche. non plus comme un point matériel. mais comme un écoulement à géométrie variable doté d'un front, ce qui est beaucoup plus réaliste. Aux forces prises en compte dans le modèle simplifiè s'ajoutent les forces de pression. On peut alimenter l'avalanche par reprise de neige en place. La topographie est beaucoup mieux respectée. On ne peut tenir compte exactement du relief. mais on peut décrire la zone parcourue par l'avalanche au moyen de profils en travers. lissés de façon à pouvoir exprimer la surface mouillée par une expression du type:

$$
s=C^{\text {te }} X \text { (hauteur }^{n}
$$

Par exemple. $\mathrm{n}=1$ pour un rectangle, $\mathrm{n}=1.5$ pour une parabole et $\mathrm{n}=2$ pour un triangle.

Quelles sont les imperfections de ce modèle? D'abord, il règne une certaine imprécision au sujet des conditions initiales. Aucun modèle de type hydraulique ne peut représenter le tout début

\footnotetext{
(*) S: densite $\times$ section $(\mathrm{hg} / \mathrm{m})$.

$P$ : densitè $\times$ section $x$ vitesse $(\mathrm{hg} / \mathrm{s})$.

$x$ : abscisse de la section.

$\psi$ : angle de la pente à rabscisse $x$.

$R$ : rayon hydraulique.

$f_{S}, f_{l}, f_{t}$ : frottements statiques, laminaires et turbulents.

$\mathrm{g}$ : acceleration de la pesanteur.
$\mathrm{h}$ : hauteur de la neige dans l'écoulement
(perpendiculairement a la pente).
$\mathrm{W}$ : vitesse du front.
$\mathrm{V}$ : vitesse de la neige du front.
$\mathrm{h}$ : hauteur de la neige en place (reprise par l'ecoulement).
} 


\section{G. BRUGNOT}

d'une avalanche, avec passage d'un état "solide" à un état "liquide". On est donc obligé de réaliser une certaine initialisation arbitraire de l'écoulement, en utilisant la formule de Voellmy. Heureusement, on peut vérifier que le choix de ces conditions de "fluidisation " n'influe guère sur le résultat ultérieur. c'est-à-dire sur les valeurs de vitesse de front et de caractéristiques géométriques de l'avalanche quelques dizaines de secondes ou quelques centaines de mètres plus tard.

Plus gênante est la difficulté de prendre en compte les variations de densité. Théoriquement celle-ci peut varier, dans des limites mal connues puisque, nous le verrons plus loin, nous n'avons pas d'observation de terrain à ce sujet. Pratiquement, il faudrait, connaissant cette loi, écrire une équation rhéologique liant la densité aux autres paramètres de l'écoulement. On aurait donc une inconnue supplémentaire et une équation supplémentaire. Pour l'instant, cette complication est sans intérêt puisqu'on ne connaît pas la rhéologie de la neige en mouvement. Mais il faut garder présent à l'esprit cette imperfection liée à une mauvaise connaissance du phénomène naturel, mauvaise connaissance à laquelle aucun ordinateur ne pourra jamais remédier.

Une question très délicate est celle de l'arrêt de l'avalanche. Elle est insoluble dans le cadre du modèle présenté puisque celui-ci considère l'avalanche comme un écoulement unidimensionnel. En pratique, une avalanche s'étale quand elle entre dans sa zone de dépôt. Ce fait ne peut être pris en compte que par un modèle bidimensionnel.

Enfin, une question complexe est celle de la prise en compte des frottements. On connait les lois de frottement et on n'a aucune raison de douter que. selon les cas, celui-ci sera solide, laminaire ou turbulent. Mais quels seuils de vitesse choisir pour déterminer le domaine de ces divers modes de frottement? Quels coefficients adopter dans chacun de ces domaines? C'est à ce genre de questions que peuvent répondre les expérimentations destinées à " caler "le modèle.

\section{Le modèle bidimensionnel}

Actuellement, toujours grâce à la collaboration entre la division "Nivologie " et la division " Hydrologie-Hydraulique fluviale", le modèle décrit plus haut a été complété par un modèle bidimensionnel qui permet de décrire l'étalement de l'avalanche dans sa zone d'arrêt et constitue un outil d'un intérêt exceptionnel pour la détermination du risque sur un cône de déjection. Ce modèle est en principe opérationnel mais avant de pouvoir servir, par exemple dans le cadre des plans des zones exposèes aux avalanches, il devra être calé à partir de nombreuses observations expérimentales.

\section{Expérimentation sur les avalanches denses. Calage des modèles}

Plus un modèle numérique est complexe, plus il a besoin d'être "calé ". Cette expression signifie que l'on doit procéder à un certain nombre d'expériences de terrain où les conditions initiales sont connues et où la dynamique du phénomène peut être mesurée.

Dans le cas des avalanches denses, nous sommes arrivés à la conclusion que la méthode la plus intéressante est la stéréophotogrammétrie (Informations techniques du C.T.G.R.E.F., 1980). Cette méthode, mise au point en U.R.S.S. (Briukhanov, 1950), revient à utiliser deux appareils photographiques synchronisés, l'intervalle séparant deux prises de vue successives étant de 1,5 secondes. Son intérêt est de permettre une "restitution" dans l'espace de l'avalanche, c'est-à-dire que le contour de l'écoulement est défini par un certain nombre de points en $x, y, z$, toutes les 1,5 secondes. Cela permet de déterminer la vitesse du tront de l'écoulement, mais aussi les lois de croissance de ses dimensions. Si l'on ne souhaite que la vitesse du front, d'autres méthodes plus simples sont utilisables: photos latérales, radar... 


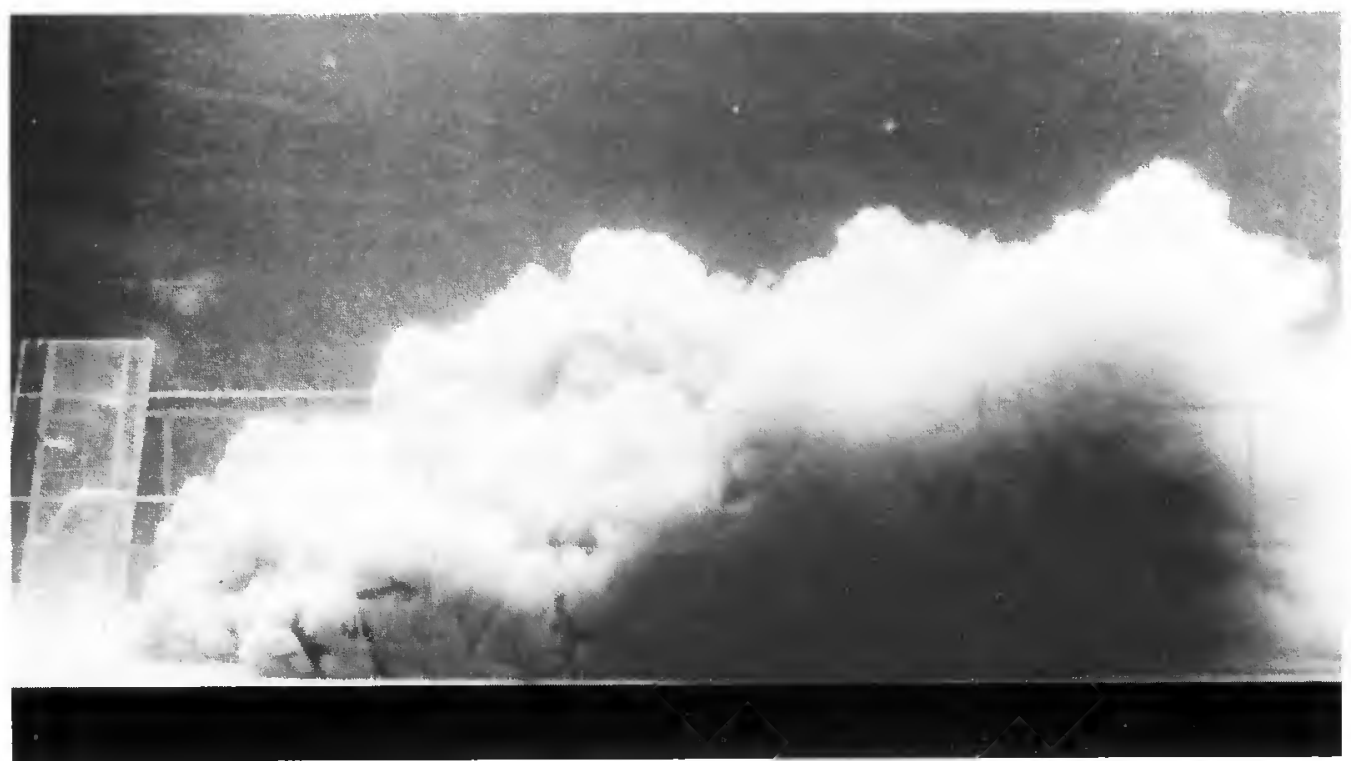

Modélisation physique d'avalanche poudreuse (bidimensionnel).

Modélisation physique d'avalanche poudreuse (tridimensionnel sur maquette au $1 / 1000^{\circ}$ ).

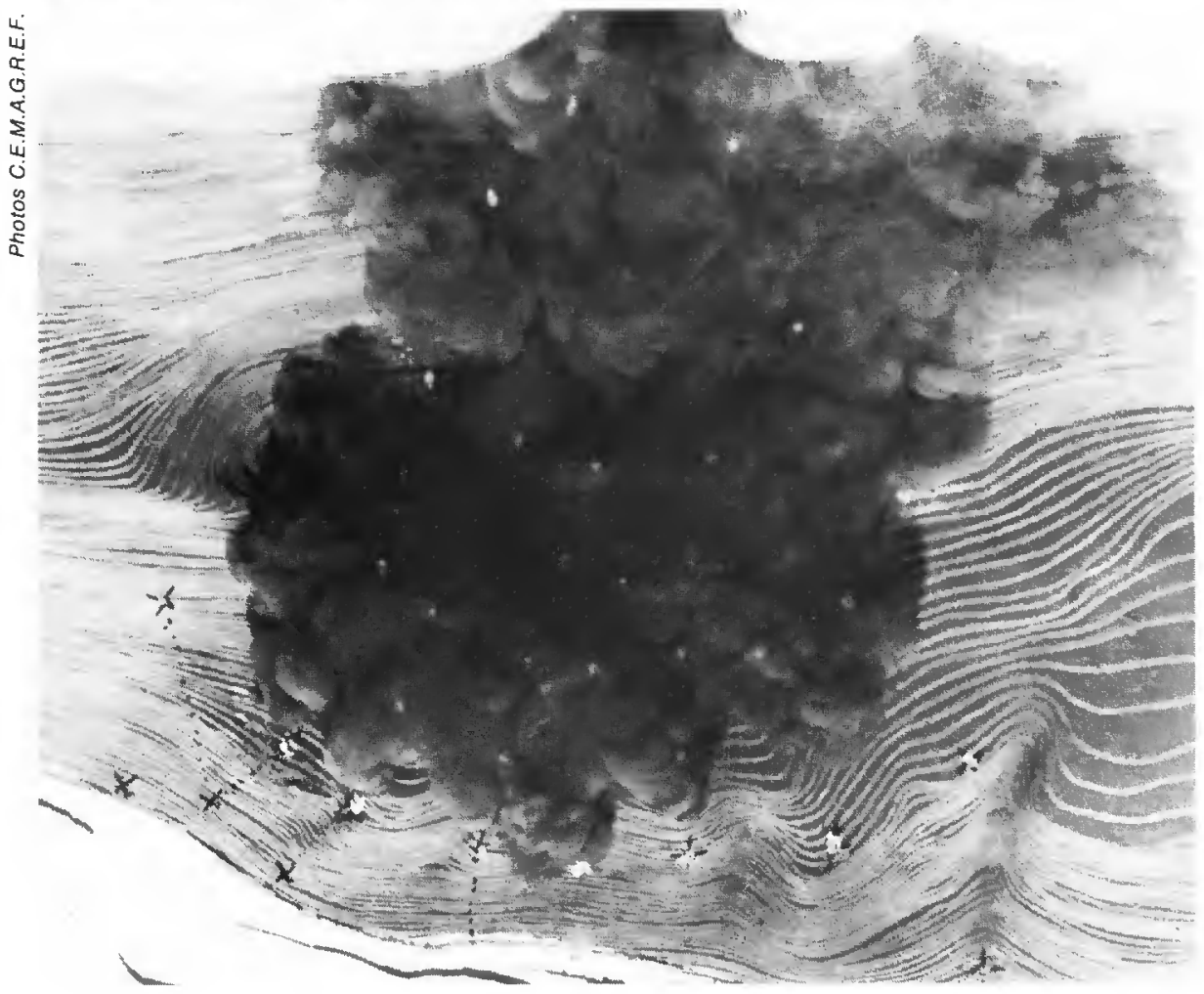




\section{G. BRUGNOT}

Pour l'instant, nous avons réalisė deux opèrations de stèrèophotogrammétrie sur des avalanches déclenchées au Col du Lautaret (Hautes-Alpes). Un deuxième site est en ètat de fonctionnement à la station de la Plagne (Savoie), mais si les avalanches attendues sont beaucoup plus importantes, les conditions d'opération sont plus délicates et nous n'avons pas encore réussi l'expérience.

A partir des avalanches citées ci-dessus, nous avons pu ajuster certains paramètres ou du moins estimer l'ordre de grandeur d'un certain nombre de coefficients. mais de nombreuses autres mesures sont nécessaires pour lever toutes les incertitudes du modèle.

Notons enfin que la question de la mesure de densitè de l'écoulement et de sa loi de variation n'a toujours reçu aucun début de solution. Nous sommes en présence d'une ènigme à la fois sur le plan théorique (difficulté de poser la loi liant la densité aux autres paramètres de l'écoulement) et pratique (difficulté de mesurer la densité). qui constituera le dernier problème à résoudre dans le domaine de la dynamique des avalanches, qu'elles soient denses ou poudreuses.

\section{Une autre approche : le modèle statistique}

Les méthodes de type Voellmy ne sont pas applicables pratiquement, elles emploient des modèles très éloignés de la réalité physique. Les modèles numèriques du type de ceux qui ont èté décrits ci-dessus nécessiteront de longues mises au point avant d'être opérationnels. Pour ces raisons, il nous semble indispensable de parler d'une approche tout à fait logique en prèsence d'un phénomène physique compliqué. l'approche statistique. A notre connaissance, le travail le plus systématique dans cette voie a été réatisé par les chercheurs norvégiens du N.G.I. (Norwegian Geotechnical Institute). C'est en partant d'un fichier de plusieurs centaines d'avalanches qu'ils ont ètabli un modèle statistique qui permet de déterminer, de façon relativement prècise, les limites extérieures d'une avalanche. L'idée de départ est de prendre comme variable à expliquer, la pente de la droite joignant le sommet de la zone de dèpart à l'extrémitè aval du culot d'avalanche. Comme variable explicative, après de multiples essais, il apparaît que le meilleur ensemble de valeurs est :

$H$ : dénivellée verticale de l'écoulement

$y^{\prime \prime}$ : rayon de courbure de l'avalanche

$\beta$ : pente moyenne de la zone canalisée

$\Theta$ : pente de la zone de dèpart

alors :

$$
\alpha=\left(0,62-0,28 \mathrm{Hy}^{\prime \prime}\right) \beta+\left(1,9 \mathrm{Hy}^{\prime \prime}-2,3\right)+0,12 \mathrm{H}
$$

$\operatorname{avec} \tilde{\sigma}=2,3^{\circ} \quad$ et $\quad R=0,95$ (Lird et Bakkehøi, 1980)

\section{MOdÉLISATION DES AVALANCHES DE NEIGE POUDREUSE}

La modelisation des avalanches de neige poudreuse est beaucoup moins ancienne que la modélisation des avalanches de neige dense. Une approche du type Voellmy/Chèzy ètait impossible car on ne pouvait pas faire d'hypothèse simple sur la croissance dimensionnelle de l'avalanche de neige poudreuse. Pour cette raison, la première approche a été, non pas numérique, mais analogique.

Stéréophotogrammétrie d'avalanches.

Couple de photos prises au Col du Lautaret (couloir $n^{\circ} 2$ ).

3 secondes séparent le couple en haut de page et le couple en bas de page. 

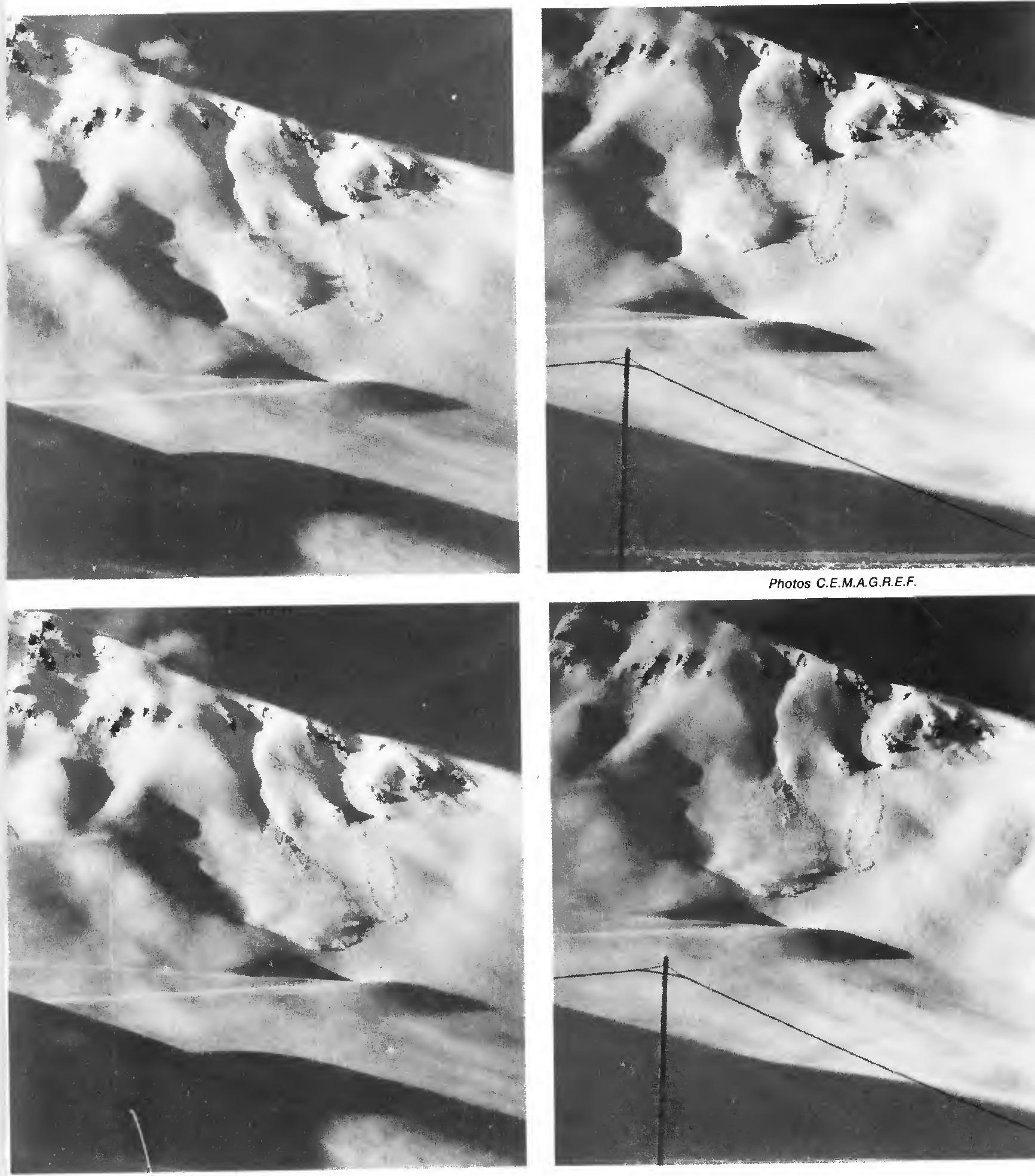


\section{G. BRUGNOT}

II apparaît en effet que la "tête" d'une avalanche de neige poudreuse est assimilable à un courant de gravité, écoulement déjà étudié car il se rencontre dans la nature dès qu'un fluide lourd s'écoule au sein d'un fluide plus léger (Michon, Godet, Bonnetille, 1955). Or, ce genre d'écoulement s'étudie assez facilement par simulation physique; pour plus de detail sur ce point, on pourra consulfer Tochon-Danguy, Hopfinger (1975). On verra qu'en réalité, la modélisation n'est possible qu'au prix de certaines simplifications, notamment une distorsion des échelles de densité. Néanmoins, on doit reconnaître que l'étude par simulation des avalanches de neige poudreuse est la première approche scientifique des courants de gravite sur forte pente; cette investigation fondamentale intéresse d'autres chercheurs, par exemple ceux qui sont confrontés au problème des avalanches sous-marines (courant de gravité par écoulement de matériaux d'origine terrestre dans la mer).

Chronologiquement, une première étude a été faite sur un canal ( $3 \mathrm{~m} \times 0,50 \mathrm{~m} \times 0,30 \mathrm{~m})$ inclinable, rempli d'eau. Dans ce canal noyé étaient injectées des solutions salines ou des solutions plus denses, comme de la baryte. II a été ainsi possible, au prix de plusieurs années d'études. de déduire un certain nombre de lois gouvernant l'évolution d'une bouffée de fluide de volume fini (Beghin et Hopfinger, 1978). Concrètement, sur une pente constante, ce genre de bouffée se diffuse, sa vitesse décroissant avec la racine carrée de la distance parcourue, tandis que ses dimensions géométriques croissent proportionnellement à cette distance, d'où une rapide décroissance concomitante de la densité.

Cefte installation de modélisation a permis également de simuler l'interaction entre l'avalanche et un ouvrage de protection. Cette phase de l'étude a été très instructive, car elle a permis de montrer à quel point le sillage d'un ouvrage de type étrave pouvait être réduit, ce qui nous a conduit à préconiser une auto-protection des structures à protéger. En effet, on peut réduire les efforts en profilant de façon adéquate un pylone, tandis qu'en le plaçant sur une étrave, on provoque une concentration locale de charge qui risque d'être fatale.

A ce stade, un certain progrès avait été réalisé, mais pour pouvoir débuter l'étude, on avait dû admettre une simplification du phénomène. Les principales hypothèses étaient l'assimilation de cet écoulement diphasique à un fluide homogène (gaz lourd), le caractère bidimensionnel et surtout l'absence d'interaction écoulement-manteau neigeux (reprise de neige). C'est probablement cefte dernière simplification qui est la plus importante et nous pensons qu'à la limite, selon que l'on admet ou non une reprise de la neige en place, on a deux types d'écoulement différents, tous deux ayant un sens physique et se rencontrant dans la nature.

On a ensuite levé assez facilement l'obstacle de la troisième dimension en réalisant un bassin relativement large $(0,80 \mathrm{~m} \times 2,50 \mathrm{~m} \times 1,50 \mathrm{~m})$. Ceci nous a permis d'adapter les lois réglant l'avancée du front et la croissance dimensionnelle. A cet ègard, on pourra consulter Beghin et Brugnof (1982).

La question très délicate de la reprise a été abordée qualitativement dans ce bassin où des expériences très intéressantes ont pû être réalisées. Le matériau qui s'est avéré le plus apte à représenter la neige en place a été la sciure de bois. Les travaux étant qualitatifs (visualisation et non modélisation), ont donné lieu, non à un article, mais à un film.

Le caractère diphasique de l'écoulement ne se manifeste que dans la phase d'arrêt de l'avalanche poudreuse qui, selon les cas, semble se diffuser ou prendre en masse. Ces phénomènes ne peuvent être expliqués qu'à partir d'un modèle diphasique, mais nous avons délibérément abandonné cet aspect de la recherche puisqu'il a été abordé par un chercheur à l'Ecole Polytechnique fédérale de Zurich. 


\section{PERSPECTIVES ET CONCLUSION}

On a pu voir que si le travail réalise dans le domaine de la modélisation des avalanches est assez important, de nombreux dèveloppements sont non seulement possibles, mais encore indispensables si l'on veut aboutir à des modèles opérationnels. Or, les crédits affectès à la recherche en matière de dynamique des avalanches sont de plus en plus réduits alors que, au fur et à mesure des progrès faits, les modèles, tant physiques que numèriques, deviennent de plus en plus coûteux.

Dans ces conditions, nous pensons que la recherche en dynamique des avalanches ne progressera que si elle met ses efforts en commun avec d'autres domaines, par exemple celui du transport de la neige par le vent et des avalanches sous-marines pour les avalanches poudreuses ou encore celui de certains écoulements de laves torrentielles pour les avalanches denses.

\section{G. BRUGNOT \\ ingènleur du G.R.E.F \\ Division "Nivologie"}

CENTRE NATIONAL DU MACHINISME AGRICOLE

DU GÉNIE RURAL, DES EAUX ET DES FORÉTS

C.E.M.A.G.R.E.F. - GRENOBLE

BP 114

38402 SAINT-MARTIN-D'HĖRES

\section{BIBLIOGRAPHIE}

BEGHIN (P.), HOPFINGER (E.J.). - Effet de la densité et de la pente sur la dynamique des avalanches poudreuses. Comptes-rendus de la $2^{e}$ Rencontre internationale sur la neige et les avalanches. Publication de l'Association internationale pour l'Etude de la Neige et des Avalanches (A.N.E.N.A.), 1978, pp. 173-187.

BEGHIN (P.), BRUGNOT (G.). - 1982. Contribution des résultats théoriques et expèrimentaux à la dynamique des avalanches de neige poudreuse. Revue de l'A.N.E.N.A., $n^{\circ} 28$, mars 1982.

BRIUKHANOV (A.V.). - [De l'ètude du mécanisme du mouvement des avalanches par la méthode stèrèophotogrammétrique (Les exigences techniques de l'appareillage)]. Sanigmi, Tachkent, 1963.

BRIUKHANOV (A.V.). - [Mécanisme du mouvement des avalanches de neige et leur ètude dans diverses conditions géographiques par une méthode de stéréophotogrammétrie spéciale]. "Neige et avalanches dans les Khibines », Moscou, 1967, pp. 269-334, texte russe.

BRUGNOT (G.), POCHAT (R.). - Numerical simulation study of avalanches. Journal of Glaciology, vol. 27. $1981, n^{\circ} 95$, pp. 77-88.

CARRY (C.), POCHAT (R.). - Modélisation d'une avalanche. Comptes-rendus de la $2^{e}$ Rencontre internationale sur la neige et les avalanches. Publication de l'Association nationale pour l'Etude de la Neige et des Avalanches (A.N.E.N.A.), 1978, pp. 189-201.

C.T.G.R.E.F. Division "Nivologie». - La stèréophotogrammètrie à cadence rapide d'avalanches. Informations techniques du C.T.G.R.E.F., cahier 39, fasc. 8, 1980, 4 p. 


\section{G. BRUGNOT}

GOFF (A.G.), OTTEN (G.F.). - [Dètermination expèrimentale de la force d'impact des avalanches]. Publication de I'Acadèmie des Sciences d'U.R.S.S., sèrie géographie et géophysique, $\mathrm{n}^{\circ} 3,1939$, texte russe.

HOPFINGER (E.J.), TOCHON-DANGUY (J.-C.). - Model study of powder snow avalanche. Journal of Glaciology, vol. $19,1977, n^{\circ} 81$, pp. 349-356.

KOULIKOVSKY (A.G.), EGLITE (M.E.). - Le problème bidimensionnel du mouvement de lavalanche de neige le long d'une pente aux caractèristiques lentement variables. Mathèmatique appliquée et mécanique, tome 37 , 1973, pp. $837-848$ (traduction disponible au C.E.M.A.G.R.E.F.).

LANG (T.E.), DAWSON (K.E.), MARTINELLI (M.). - Numerical simulation of avalanche flow. Rocky Mountain forest and range experiment station. Research paper, R.M.-205, 1979, 51 p.

LIED (K.), BAKKEHФI. - Empirical calculations of snow avalanche distance based on topographic parameters. Journal of Glaciology, vol. 26, $n^{\circ} 94,1980, \mathrm{pp} .165-177$.

MICHON (X.), GODET (J.), BONNEFILLE (R.). - Etude théorique et expérimentale des courants de gravité. Paris: Laboratoire National d'Hydraulique, 1955, 3 vol.

PERLA (R.I.), MARTINELLI (M.). - Avalanche randbook. - U.S.D.A. Agriculture handbook, $n^{\circ} 489,1976$, V, $238 \mathrm{p}$.

PERLA (R.I.). - Avalanche release, motion and impact. In : Dynamics of snow and ice masses. Academic Press, 1980, pp. 397-456.

SAMOYLOV (V.A.). - [Prise de vue stéréophotogrammétrique d'une avalanche en mouvement dans les Khibines]. Matériaux de recherche glaciologique, tome 28, 1976, pp. 128-133 (en russe).

TOCHON-DANGUY (J.-C.), HOPFINGER (E.J.). - Simulation of the dynamics of powder avalanches. - In : Snow mechanics. Colloque de Gindelwald. International association of scientific hydrology, publication 114,1975, pp. $369-380$

VAN WIJK (N.C.). - Photogrammetry applied to avalanche study. Journal of glaciology, vol. $6,1967, \mathrm{n}^{\circ} 48$, pp. $917-933$.

VOELLMY (A.). - Ueber die Zerstörungskraft von Lawinen. Schweizerischen Bauzeitung, vol. 73, 1955, $\mathrm{n}^{\text {os }} 12,15$, $17,19,37$. 